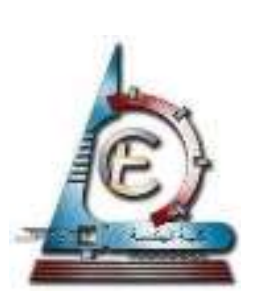

Journal of Engineering Sciences

Assiut University

Faculty of Engineering

Vol. 43

No. 4

July 2015

PP. $472-489$

\title{
DESIGN OF ROBUST PID CONTROLLERS \\ FOR FIRST-ORDER PLUS TIME DELAY SYSTEMS \\ BASED ON FREQUENCY DOMAIN SPECIFICATIONS
}

\author{
Noha Medhat Darwish \\ Electrical Engineering Dept., Faculty of Engineering, Assiut University, Assiut, Egypt \\ (Received 6 May 2015; Revised 12 June 2015; Accepted 19 July 2015)
}

\begin{abstract}
This paper considers a design method for PID controllers to achieve the robustness to the uncertainty of the time delay for the first-order plus time delay system (FOPTD). Initially, the stabilizing regions of the PID controller gains are determined by a graphical stability method. Then, we specify two simultaneous design specifications: gain margin and phase crossover frequency. These specifications give a set of stabilizing PID controllers. To get a unique PID controller, we introduce an additional constraint which is finding the smallest absolute value of the slope of the open-loop system magnitude at the specified phase crossover frequency. The obtained PID controller is located in the stability region, and also robust to system time delay variation due to the proposed constraint.
\end{abstract}

Keywords: First-Order Plus Time Delay (FOPTD) systems, Gain margin, Proportional-IntegralDerivative (PID) controllers, Robustness, Stabilizing region.

\section{Introduction}

Over $90 \%$ of the process control and industrial applications are controlled by the proportional-integral-derivative (PID) controllers $[1,2]$. The PID controller has a simple structure and gives a good performance in the closed-loop response.

Practically, there are several methods for tuning of PID gains such as Ziegler-Nichols methods [3]. These methods may give a satisfactory closed-loop response, but they cannot give any information on the stabilizing region of these gains.

The primary aim of the controller design is to maintain the stability of the control system. There are several methods for computing the stabilizing PID controllers for delay-free linear time invariant systems [4-8]. However, many of industrial systems have time delay, so the stable plant that has an S-shaped step response is modeled as first- or second-order plus time delay models [1].

Several methods for computing the stabilizing PID controllers for the first-order plus time delay (FOPTD) systems are proposed by the authors of [4, 6, 9-11]. In [4, 6, and 10], an extension of the Hermite-Biehler Theorem based on Pontryagin results [12] was investigated to determine all the stabilizing PID controllers. An alternative way is proposed 
in $[4,6]$ using the generalized Nyquist stability criterion. In [9], the stabilizing regions of the PID gains are obtained by the graphical stability criterion for the FOPTD systems.

As the stability is an important goal for designing the PID controllers, but it is not enough requirement to get a satisfactory response behavior (robustness and performance). Robust control is an area of intensive research. It can be achieved using gain and phase margin specifications [9, 13-15] or closed-loop sensitivity constraint. The sensitivity constraint is similar with the gain and phase margin specifications as they have a direct relation $[1,16]$. In [13], simple tuning rules for designing PID controller are obtained to satisfy optimal gain and phase margins. In [14], the robustness is achieved by gain and phase margins while the closed-loop performance is achieved by bandwidth and maximum amplitude ratio. The limitation of this work is the nonlinear optimization problem which needs numerical solution. In $[9,15]$, the robust design is proposed by achieving the pre-specifications of phase margin and gain crossover frequency in addition to the flat phase tuning constraint. The obtained controller has two properties: first, its gains are located in the stabilizing region. Second, it is robust to a bounded variation in the system gains, which include the uncertainty of the plant steady-state gain and the overall variations of the controller gains.

Uncertainty in dc gain of the model is important but in dealing with time delay plants, uncertainty in time delay is more important and critical. In this paper, we consider the problem of time delay uncertainty by proposing a method to design PID controllers to achieve the pre-specifications of gain margin, phase crossover frequency, and a minimum absolute value of the derivative of the open-loop system magnitude with respect to the frequency at the specified phase crossover frequency based on the FOPTD systems. This minimum derivative ensures the robustness to a bounded variation in the system time delay and makes the closed-loop system more robust than the method proposed by the authors of [13]. For a specified gain margin, choosing a value for the phase crossover frequency affects on the location of PID gains in the stabilizing region, so the closed-loop robustness and performance can be adjusted.

As a starting point, all stabilizing PID controllers are determined using a graphical stability method which is a simple algorithm. Then, we obtain the three dimension relative stability regions that satisfy the designed gain margin. It is necessary to determine these stabilizing regions because the first aim of the proposed method is to get a set of stabilizing PID controllers that guarantee two simultaneous design specifications: gain margin and phase crossover frequency. From this set, we search for the smallest rate of change of the open-loop magnitude at the specified phase crossover frequency point $w_{c}$ to get a unique PID controller which is the second aim.

The contributions of this paper can be summarized as follows:

(1) It considers a design method that makes the closed-loop system more robust than the method proposed in [13] due to the smallest slope constraint at the specified phase crossover frequency.

(2) It treats the problem of time delay uncertainty which was not considered in $[9,15]$.

(3) It proposes a simple approach that provides a satisfactory response behavior (performance and robustness) that it does not require numerical solution for the nonlinear optimization as in [14]. 
This paper is organized as follows: In the next section, stabilizing and robust PID controller design is presented. Then, an illustrative example is given. Conclusion is provided in the last section.

\section{Stabilizing and robust PID controller design for FOPTD systems}

\subsection{FOPTD systems and PID controller}

The design method proposed in this paper is based on the FOPTD systems which is mathematically described by the transfer function,

$$
G(s)=\frac{K}{(1+T s)} e^{-L s}
$$

Consider the feedback control system as shown in Fig. 1 with a gain-phase margin tester [17], where $G(s)$ is the FOPTD plant and $G_{c}(s)$ is the designed PID controller with the transfer function,

$$
G_{c}(s)=\frac{K_{d} s^{2}+K_{p} s+K_{i}}{s}
$$

where, $K_{d}$ is the derivative gain, $K_{p}$ is the proportional gain, and $K_{i}$ is the integral gain.

The gain-phase margin tester is represented by the frequency independent transfer function $A_{m} e^{-j \theta_{m}}$. It provides information for plotting the boundaries of all stabilizing PID controller gains that achieve specified gain and phase margins [18].

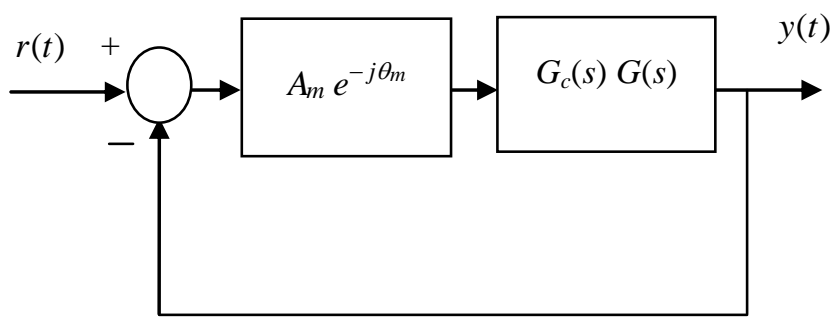

Fig. 1. Feedback control system with gain-phase tester.

For design specification on the gain margin $A_{m}$, we set $\theta_{m}=0$, and for design specification on the phase margin $\theta_{m}$, we set $A_{m}=1$.

2.2. Stabilizing region of the PID gains

From Fig. 1, the closed-loop transfer function is:

$$
T(s)=\frac{A_{m} e^{-j \theta_{m}} G_{c}(s) G(s)}{1+A_{m} e^{-j \theta_{m}} G_{c}(s) G(s)}
$$

So, the characteristic polynomial will be as follows:

$$
\delta(s)=1+A_{m} e^{-j \theta_{m}} G_{c}(s) G(s)=0
$$


By substituting equations (1) and (2) into equation (4), we obtain

$$
\begin{aligned}
\delta(s) & =s(T s+1)+K A_{m} e^{-j \theta_{m}} e^{-L s}\left(K_{d} s^{2}+K_{p} s+K_{i}\right) \\
& =T s^{2}+s+K A_{m} e^{-j \theta_{m}} e^{-L s}\left(K_{d} s^{2}+K_{p} s+K_{i}\right)=0
\end{aligned}
$$

Determining all the stabilizing PID controllers for the FOPTD plant is a primary aim in controller design. The closed-loop system is stable if all the roots locations of the polynomial in equation (5) with $A_{m}=1$ and $\theta_{m}=0^{\circ}$ are in the left-half of the s-plane. The location of these roots depends on the PID gains which are $K_{d}, K_{p}$, and $K_{i}$. The boundaries of these gains can be determined by the infinity root boundary (IRB), real root boundary (RRB) and complex root boundary (CRB) [18-20].

(i) IRB: $\delta(s=\infty)=0$, so we get a boundary on $K_{d}$ as:

$$
K_{d}= \pm \frac{T}{K}
$$

This boundary can be obtained as follow:

Put $\delta(s=\infty)=0$, so the coefficient of $s^{2}$ will be equal to zero,

$T+K A_{m} e^{-j \theta_{m}} e^{-L s} K_{d}=0$

Put $\left(A_{m}=1\right.$ and $\left.\theta_{m}=0^{\circ}\right)$, we obtain:

$T+K K_{d} e^{-L s}=0$

$e^{-L s}$ can be approximated by a first-order or second-order Pade approximation.

For first-order Pade approximation:

$e^{-L s}=\frac{2-L s}{2+L s}$, then $\left.K_{d}\right|_{s=\infty}=-\frac{T}{K}$

For second-order Pade approximation:

$$
e^{-L s}=\frac{12-6 L s+L^{2} s^{2}}{12+6 L s+L^{2} s^{2}}, \text { then }\left.K_{d}\right|_{s=\infty}=\frac{T}{K}
$$

so we get a boundary on $K_{d}$ as:

$$
K_{d}= \pm \frac{T}{K}
$$

The same result can be obtained using third-order and fourth-order Pade approximations. This means that $K_{d}$ will have positive and negative values.

(ii) RRB: $\delta(s=0)=0$, so we get a boundary on $K_{i}$ as:

$$
K_{i}=0
$$


(iii) $\mathrm{CRB}: \delta(s=j w)$, the resulting polynomial will be as follows:

$$
\begin{aligned}
& \delta(j w)=\left(-T w^{2}+j w\right)+K A_{m} e^{-j\left(w L+\theta_{m}\right)}\left(-w^{2} K_{d}+j w K_{p}+K_{i}\right)=0 \\
& =\left(-T w^{2}+j w\right)+K A_{m}\left(\cos \left(w L+\theta_{m}\right)-j \sin \left(w L+\theta_{m}\right)\right)\left(-w^{2} K_{d}+j w K_{p}+K_{i}\right)=0
\end{aligned}
$$

The real part $R(w)$ and the imaginary part $I(w)$ are as follows:

$$
\begin{gathered}
R(w)=-T w^{2}+K A_{m}\left(w K_{p} \sin \left(w L+\theta_{m}\right)+\left(-w^{2} K_{d}+K_{i}\right) \cos \left(w L+\theta_{m}\right)\right)=0 \\
I(w)=w+K A_{m}\left(-\left(-w^{2} K_{d}+K_{i}\right) \sin \left(w L+\theta_{m}\right)+w K_{p} \cos \left(w L+\theta_{m}\right)\right)=0
\end{gathered}
$$

It is clear that from equations (7) and (8) both the real as well as the imaginary parts of $\delta(j w)$ depend on $K_{d}, K_{p}$, and $K_{i}$, which causes difficulties when trying to find the range of these gains that make the system stable. To overcome this problem, we will now multiply the characteristic polynomial of equation (6) by $e^{j\left(w L+\theta_{m}\right)}$ for which only the imaginary part of the resulting polynomial depends on $K_{p}$ only.

$$
\begin{aligned}
& \text { Multiply equation (6) by } e^{j\left(w L+\theta_{m}\right)} \\
& e^{j\left(w L+\theta_{m}\right)} \delta(j w)=e^{j\left(w L+\theta_{m}\right)}\left(-T w^{2}+j w\right)+K A_{m}\left(-w^{2} K_{d}+j w K_{p}+K_{i}\right)=0 \\
& =\left(\cos \left(w L+\theta_{m}\right)+j \sin \left(w L+\theta_{m}\right)\right)\left(-T w^{2}+j w\right)+K A_{m}\left(-w^{2} K_{d}+j w K_{p}+K_{i}\right) \\
& =\operatorname{Re}(w)+j \operatorname{Im}(w)=0
\end{aligned}
$$

where $\operatorname{Re}(w)$ is the real part and $\operatorname{Im}(w)$ is the imaginary part as follows:

$$
\begin{aligned}
& \operatorname{Re}(w)=-T w^{2} \cos \left(w L+\theta_{m}\right)-w \sin \left(w L+\theta_{m}\right)-K A_{m} w^{2} K_{d}+A_{m} K K_{i}=0 \\
& \operatorname{Im}(w)=w \cos \left(w L+\theta_{m}\right)-T w^{2} \sin \left(w L+\theta_{m}\right)+K A_{m} w K_{p}=0
\end{aligned}
$$

Equating the real part and the imaginary part to zero, we obtain:

$$
\begin{aligned}
K_{p} & =\frac{T w \sin \left(w L+\theta_{m}\right)-\cos \left(w L+\theta_{m}\right)}{K A_{m}} \\
K_{i} & =\frac{w \sin \left(w L+\theta_{m}\right)+T w^{2} \cos \left(w L+\theta_{m}\right)}{K A_{m}}+w^{2} K_{d}
\end{aligned}
$$

For each fixed value of $K_{d}$, we can determine the values of $K_{p}$ and $K_{i}$ as $w$ changes from zero to infinity using equations (12), (13) and plot the curve of $K_{i}$ versus $K_{p}$ that describes these values. This curve divides the two-dimension space $\left(K_{p}-K_{i}\right)$ into stable and unstable regions by $\mathrm{RRB}$ and $\mathrm{CRB}$. The stabilizing region can be determined by choosing an arbitrary point in each region. Mathematically, equation (12) can give positive and negative values of $K_{p}$ that stabilize the FOPTD system depending on the values of $w$.

By sweeping over all values of $K_{d} \in[-T / K, T / K]$, we can determine all the threedimension stabilizing regions for the PID controller gains. 


\subsection{Stabilizing PID controllers that guarantee two frequency domain specifications}

The first aim of the design method is to determine the relative stability point on the $\left(K_{p}-K_{i}\right)$ curve with a fixed value of $K_{d}$ that guarantees two frequency domain specifications which are gain margin $A_{m}\left(\theta_{m}=0\right)$ and phase crossover frequency $w_{c}$.

This point can be obtained as follows:

(i) Setting $2 \leq A_{m} \leq 5$ [13], $\theta_{m}=0$ in equations (12), (13) and finding the $\left(K_{p}-K_{i}\right)$ curve with each fixed value of $K_{d} \in[-T / K, T / K]$ as $w$ changes from zero to $w_{0}$, where $w_{0}$ is the maximum frequency satisfying the specified gain margin with fixed $K_{d}$. Of course this curve is always inside the stabilizing region obtained in section 2.2 at the same $K_{d}$. Since $A_{m}=$ spesified value and $\theta_{m}=0$, all $w \in\left(0, w_{0}\right]$ that satisfy equation (6) will be considered as phase crossover frequencies for the feedback control system in Fig. 1 where,

$$
\left|A_{m} G_{c}(j w) G(j w)\right|=1
$$

This means that $w_{0}$ is the maximum feasible $w_{c}$ with each fixed $K_{d}$ and $A_{m}$.

(ii) For a specified phase crossover frequency $w_{c}$, and a fixed value of $K_{d} \in[-T / K, T / K]$, we can determine a unique point on the $\left(K_{p}-K_{i}\right)$ curve.

Repeating (i) and (ii) with all values of $K_{d} \in[-T / K, T / K]$, a line in three-dimension gain space can be determined. Each point on this relative stability line guarantees the two specifications $A_{m}$ and $w_{c}$.

We can also draw a curve that represents the $\left(w_{0}-K_{d}\right)$ relation and then the upper boundary $w_{0 \max }$ will be known. So, with a fixed gain margin, the gains of the PID controller can be constructed depending on a chosen value of the phase crossover frequency $w_{c}$ inside the interval $\left(0, w_{0 \max }\right]$.

\subsection{Determining the unique robust PID controller with a magnitude slope constraint}

In this section, an additional specification is introduced to get unique PID controller gains, by making a condition on the absolute value of the slope of the open-loop system magnitude at the phase crossover frequency point $w_{c}$.

The second aim of the design method is to make the feedback control system in Fig. 1 robust to a bounded variation in the system time delay. This can be achieved by finding the smallest rate of change of the open-loop magnitude at the specified phase crossover frequency point $w_{c}$. This means that the change in the gain margin is small for any variation in the system time delay $L$ in a limited range. By observing the phase curve of the open-loop transfer function:

$\varnothing(w)=-w L-\tan ^{-1} w T-90+\tan ^{-1}\left(w K_{p} /\left(K_{i}-w^{2} K_{d}\right)\right)$, we can conclude that: Any bounded variation in the time delay affects directly on the open-loop phase curve by moving it up or down, since the phase equation is function of $L$ and as a result, the value of 
the gain margin will be changed. This change is due to the variation in the phase crossover frequency value. If the absolute value of the slope of the magnitude curve at the specified phase crossover frequency $w_{c}$ is small, so the variation in the gain margin value is small and the closed-loop step response is still have a good performance, and the robustness property is achieved.

Equate equations (7) and (8) and put $\theta_{m}=0$, we obtain

$$
\begin{aligned}
& K A_{m}\left(\left(-w^{2} K_{d}+K_{i}\right) \cos (w L)+w K_{p} \sin (w L)-w K_{p} \cos (w L)+\left(-w^{2} K_{d}+K_{i}\right) \sin (w L)\right) \\
& \quad=w^{2} T+w
\end{aligned}
$$

Then the gain margin equation will be as follows:

$$
A_{m}=\frac{w^{2} T+w}{K\left(\left(-w^{2} K_{d}+K_{i}\right)(\cos (w L)+\sin (w L))+w K_{p}(\sin (w L)-\cos (w L))\right.}
$$

Since $\left|G G_{c}\right|=1 / A_{m}$, then

$$
\left|G(j w) G_{c}(j w)\right|=\frac{K\left(\left(-w^{2} K_{d}+K_{i}\right)(\cos (w L)+\sin (w L))+w K_{p}(\sin (w L)-\cos (w L))\right.}{w^{2} T+w}
$$

From equation (17), we can get,

$$
\left|\frac{d \mid G(j w) G_{c}(j w)}{d w}\right|=\left|\frac{K(E 1 \cos (w L)+E 2 \sin (w L))}{\left(w^{2} T+w\right)^{2}}\right|
$$

where,

$$
\begin{aligned}
& E 1=-\left(w^{2}+w^{4} L T+w^{3} L\right) K_{d}+\left(w^{3} L T+w^{2}(L+T)\right) K_{p}+\left(w^{2} L T+w(L-2 T)-1\right) K_{i}, \\
& E 2=\left(-w^{2}+w^{4} L T+w^{3} L\right) K_{d}+\left(w^{3} L T+w^{2}(L-T)\right) K_{p}-\left(w^{2} L T+w(L+2 T)+1\right) K_{i}
\end{aligned}
$$

As discussed in section 2.3, all PID gains points on the relative stability line that guarantee the two specifications $A_{m}$ and $w_{c}$ will be substituted in equation (18) and the values of this equation are computed at these gains. Then we search among these values for the smallest one. The resulting PID controller makes the closed-loop system in Fig. 1 robust to bounded system time delay variation. In some cases, the designed PID controller may give phase margin greater than 70 degree (very slow closed-loop step response) or less than 30 degree (oscillatory closed-loop step response), so we need to search for the smallest magnitude slope with a condition on the phase margin range to be $30^{\circ}<\theta_{m}<70^{\circ}$. Although in this case, the slope is not the minimum value which means the degree of robustness is reduced, but the robustness is still preserved with a good performance. 


\section{An illustrative example}

Consider a FOPTD system described by the transfer function $G(s)=\frac{e^{-0.3 s}}{(s+1)}$

We will apply the proposed method to get the robust PID controller as follows:

1. Determine all the three-dimension stabilizing regions for the PID controller gains using equations (12) and (13) $\left(A_{m}=1\right.$ and $\left.\theta_{m}=0^{\circ}\right)$ by sweeping over all values of $K_{d} \in[-1,1]$. By choosing $K_{d}=0.1$, we can plot the stabilizing curve of $K_{i}$ versus $K_{p}$ as shown in Fig. 2. The all stabilizing regions are shown in Fig. 3.

2. Setting $A_{m}=3$ and $\theta_{m}=0^{\circ}$ in equations (12), (13) and finding the relative stability curve $K_{i}$ versus $K_{p}$ with each fixed value of $K_{d} \in[-0.33,0.33]$ as $w$ changes from zero to $w_{0}$, where $w_{0}$ is the maximum frequency satisfying the specified gain margin with each value of $K_{d}$ as shown in Fig. 4 . For $K_{d}=0.1$, the relative stability curve is shown in Fig. 2 , where $w_{0}=6.721 \mathrm{rad} / \mathrm{sec}$. Of course this curve is inside the stabilizing region $\left(A_{m}=1\right)$. The curve that represents the $\left(K_{d}-w_{0}\right)$ relation is shown in Fig. 5 . From this curve, the upper boundary $w_{0 \max }$ will be known, which is $10.201 \mathrm{rad} / \mathrm{sec}$.

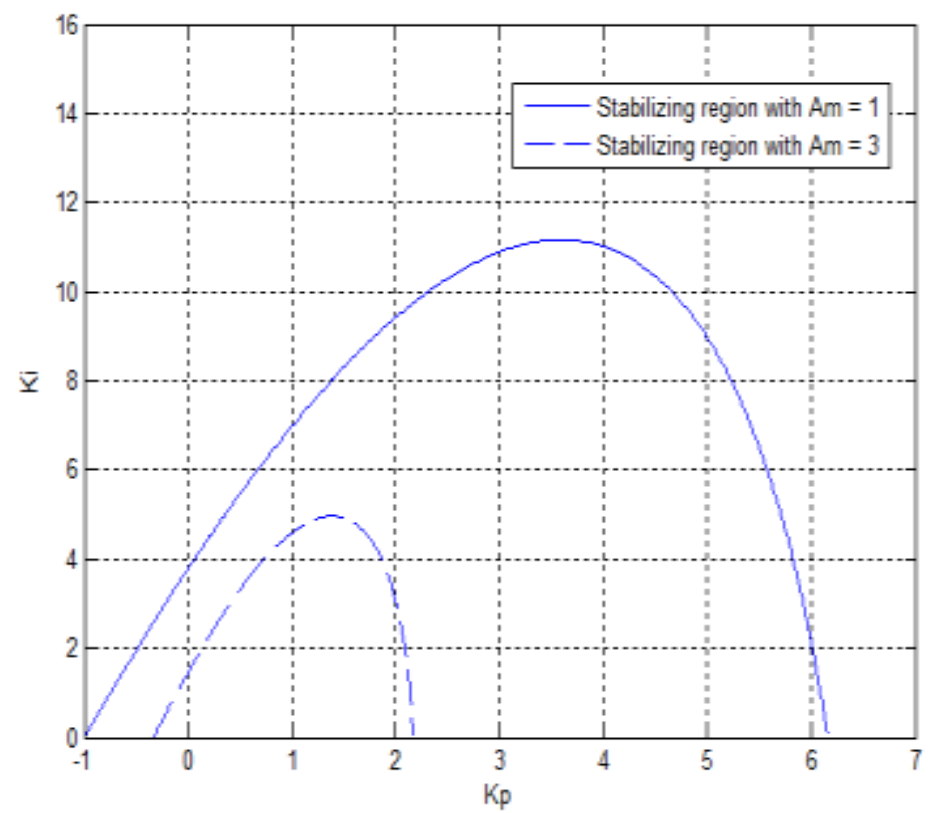

Fig. 2. The stabilizing regions with $K_{d}=0.1$ 


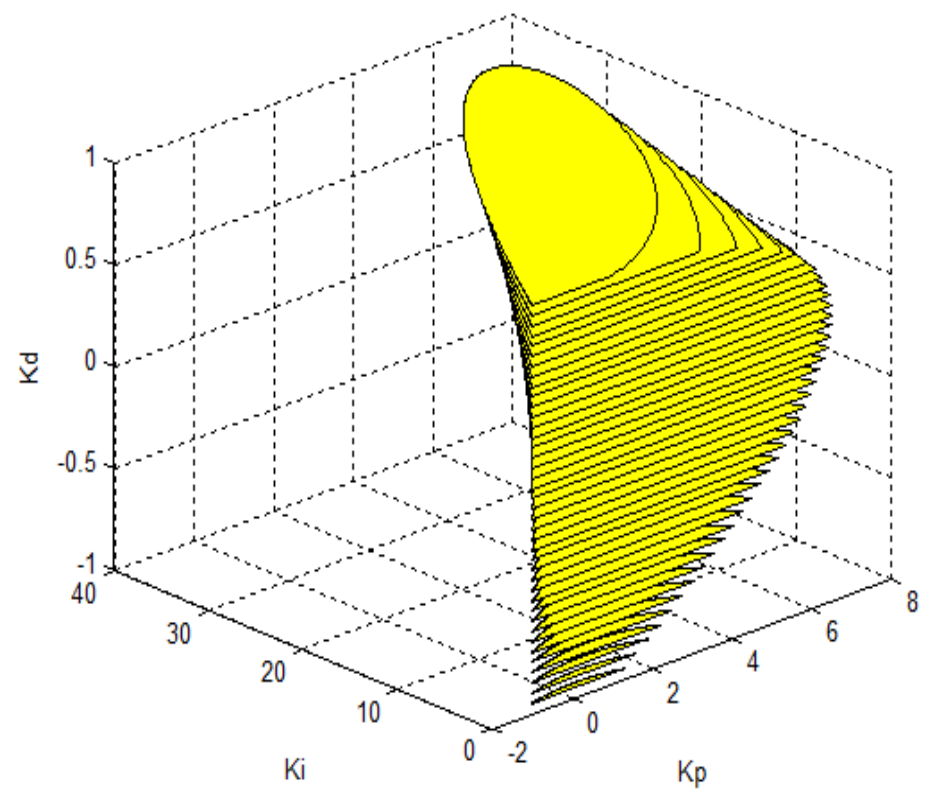

Fig. 3. All the stabilizing regions of $\left(K_{p}, K_{i}, K_{d}\right)$ values.

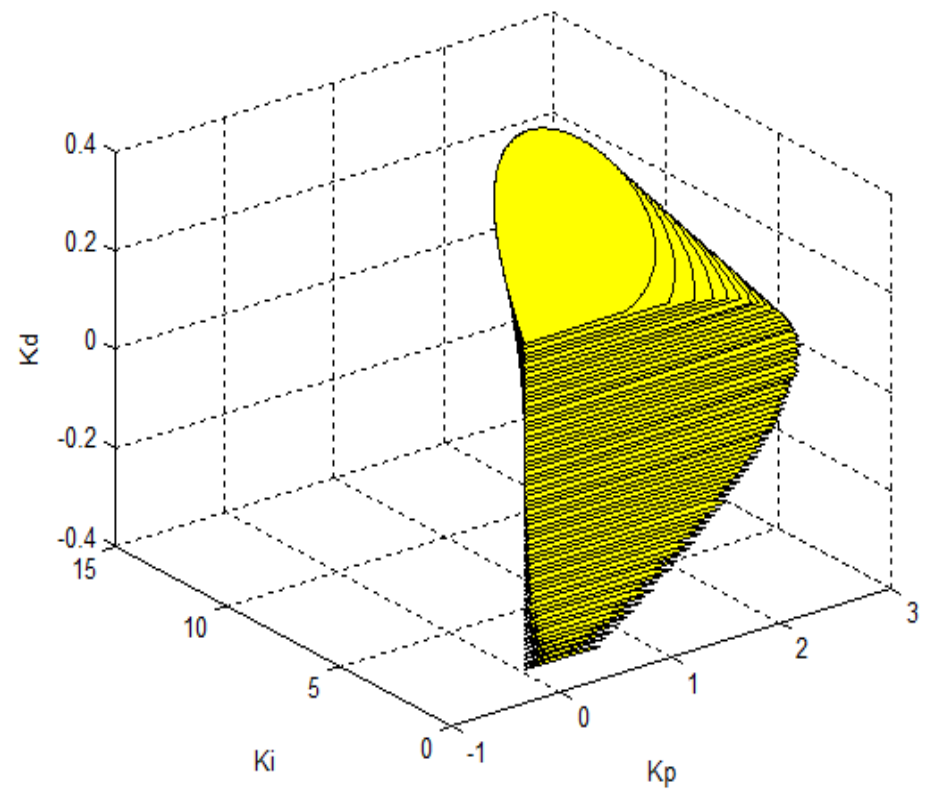

Fig. 4. The relative stability regions $\left(K_{p}, K_{i}, K_{d}\right)$ values with $A_{m}=3$. 


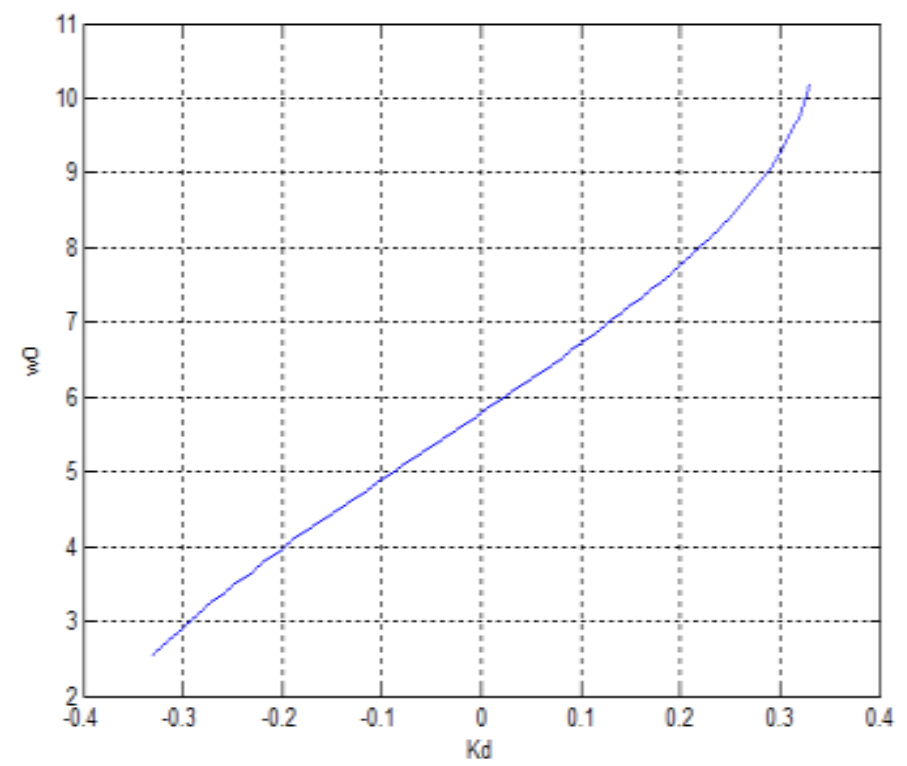

Fig. 5. The curve that represents $\left(K_{d}-w_{0}\right)$ relation.

3. Since there are several PID controllers for each fixed value of $K_{d}$ and $A_{m}$ as shown in Fig. 2., we chose any value of $K_{d} \in[-0.33,0.33]$, e.g. $K_{d}=0.1$, and $A_{m}=3$. Given a value for the phase crossover frequency e.g. $w_{c}=4 \mathrm{rad} / \mathrm{sec}$, where $w_{c} \in\left(0, w_{0 \max }\right]$, we can determine a unique point $\left(K_{p}=1.117, K_{i}=4.7687\right)$ marked by a big dot symbol on the $\left(K_{p}-K_{i}\right)$ relative stability curve (that is drawn before in Fig. 2.) as shown in Fig. 6.

$$
G_{c 0}(s)=\frac{0.1 s^{2}+1.117 s+4.7687}{s}
$$

$G_{c 0}(s)$ is a one of many PID controllers that guarantee the two specifications $A_{m}=3$ and $w_{c}=4 \mathrm{rad} / \mathrm{sec}$, but it does not guarantee the robustness. We will use $G_{c 0}(s)$ to compare its performance with the performance of robust PID controller (step 4) as it presented on the page after the next one.

By sweeping over all values of $K_{d} \in[-0.33,0.33]$, a line in three-dimension gain space can be determined as shown in Fig. 7. Each point on this relative stability line guarantees the two specifications $A_{m}=3$ and $w_{c}=4 \mathrm{rad} / \mathrm{sec}$.

4. Finding the robust PID controller (proposed PID) by substituting all PID gains points on the relative stability line that guarantee $A_{m}=3$ and $w_{c}=4 \mathrm{rad} / \mathrm{sec}$ in equation (18), and then we search for the smallest value. This point $\left(K_{d}=-0.11\right.$, $K_{p}=1.117, K_{i}=1.4238$ ) is marked by a big dot symbol as shown in Fig. 7 . The resulting controller is: 


$$
G_{c-\text { proposed }}(s)=\frac{-0.11 s^{2}+1.117 s+1.4238}{s}
$$

The bode plots of the open-loop transfer functions with $G_{c 0}(s)$ and the robust PID controller $G_{c-p r o p o s e d}(s)$ are shown in Fig. 8. It is clear that, the absolute value of the slope of the magnitude curve using $G_{c \text {-proposed }}(s)$ at the specified phase crossover frequency $w_{c}=4$ $\mathrm{rad} / \mathrm{sec}$ is smaller than that using $G_{c 0}(s)$. It is also clear that, the desired gain margin value is achieved using both PID controllers $(20 \log 3=9.54 \mathrm{~dB})$ at $w_{c}=4 \mathrm{rad} / \mathrm{sec}$.

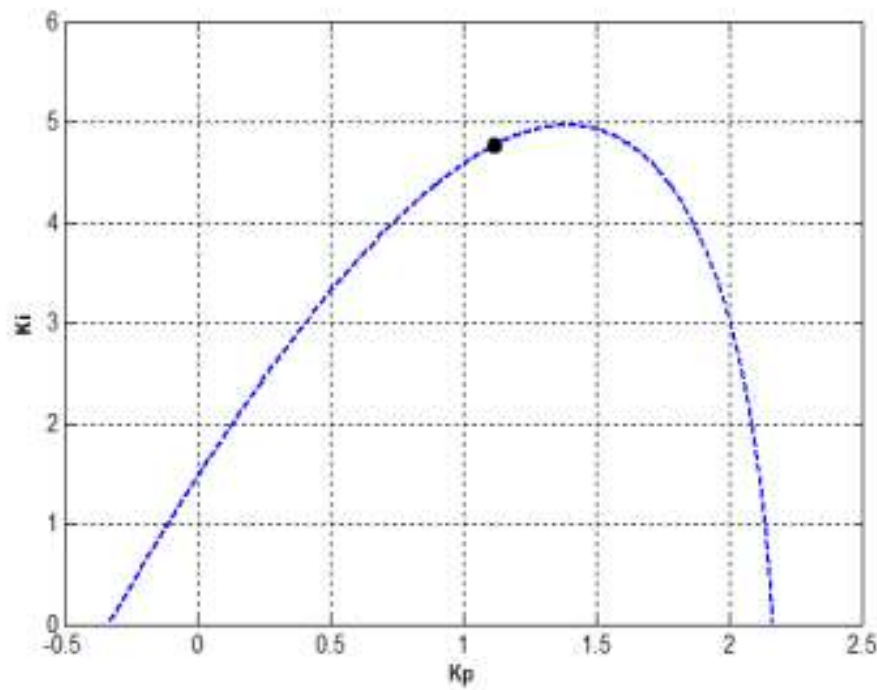

Fig. 6. The $\left(K_{p}, K_{i}\right)$ point with $K_{d}=0.1$ that satisfies $A_{m}=3$ and $w_{c}=4 \mathrm{rad} / \mathrm{sec}$.

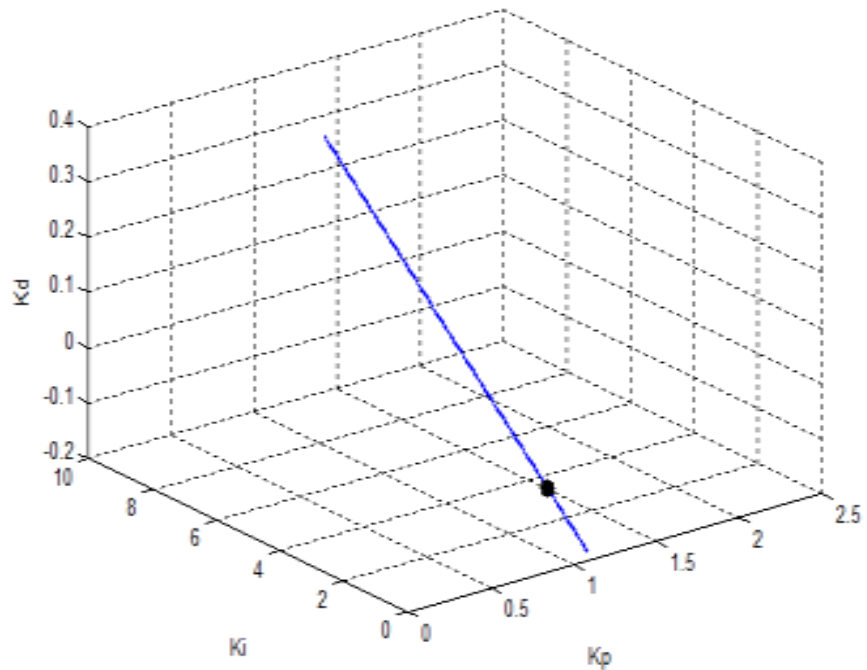

Fig. 7. The smallest magnitude slope point on the relative stability line with $A_{m}=3$ and $w_{c}=4 \mathrm{rad} / \mathrm{sec}$. 


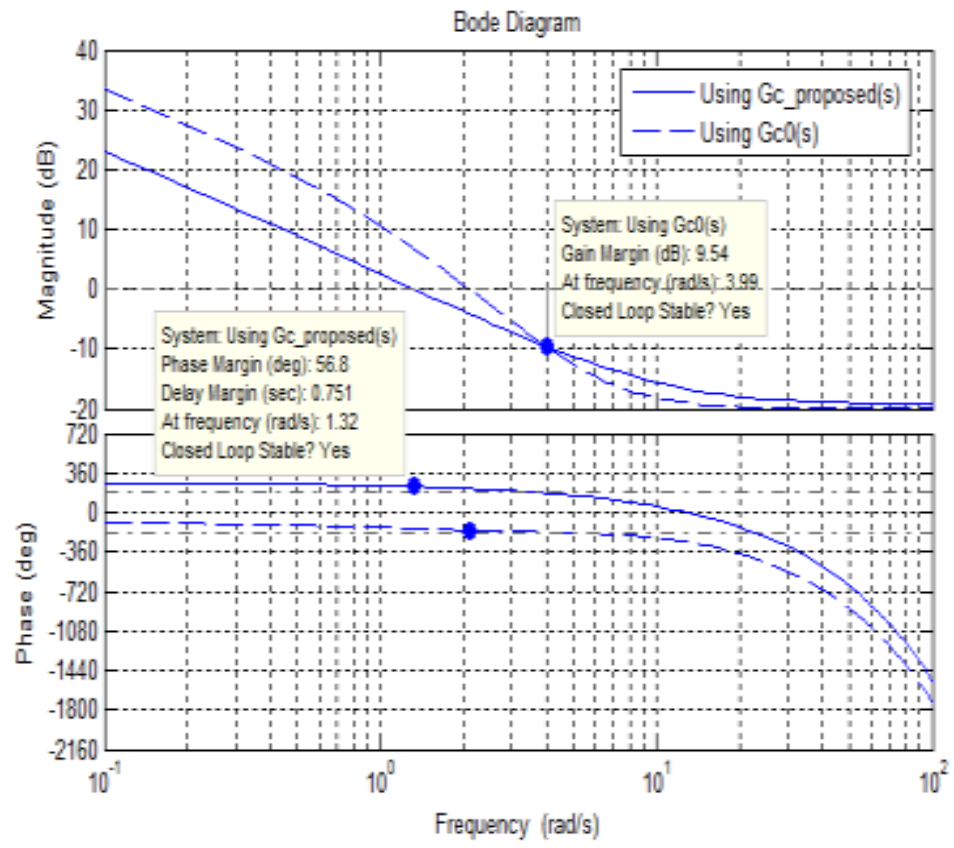

Fig. 8. The bode plots of the open-loop transfer functions.

The unit step response of the closed-loop system using the proposed PID controller $G_{c-p r o p o s e d}(s)$ with different time delay variations is shown in Fig. 9. It is clear that the effect of the variation in the system time delay by $\pm 20 \%$ on the step response is small. This means that the robustness is achieved.

The unit step response of the closed-loop system using another PID controller $G_{c 0}(s)$ with different system time delay variations is shown in Fig. 10. It is shown that the effect of the variation in the time delay by $\pm 20 \%$ on the step response is large and the robustness is not achieved.

A comparison is made with two different methods. We computed the PID controllers using the Ziegler-Nichols frequency response method [1,3] and the PID controller using the tuning rules for setpoint (ISE-GPM-setpoint) [13].

Ziegler-Nichols method is based on a simple characterization of the process dynamics. The design is based on knowledge of the point on the Nyquist curve of the process transfer function $G(s)$ where the Nyquist curve intersects the negative real axis. This point is characterized by the parameters $K_{u}$ and $T_{u}$ which are called the ultimate gain and the ultimate period. Ziegler-Nichols gives simple formulas for the gains of the PID controller in terms of the ultimate gain and the ultimate period.

The parameters of Ziegler-Nichols frequency response method will be as follows:

$k_{u}=5.8902, w_{u}=5.8047 \mathrm{rad} / \mathrm{sec}$,

and $T_{u}=2 \pi / w_{u}=1.0824 \mathrm{sec}$

The PID controller gains are: 


$$
\begin{aligned}
K_{p} & =0.6 k_{u}=3.5341, \\
K_{i} & =K_{p} / T_{i}=6.5299, \text { where } T_{i}=0.5 T_{u}=0.5412, \\
K_{d} & =K_{p} T_{d}=0.4782, \text { where } T_{d}=0.125 T_{u}=0.1353
\end{aligned}
$$

The unit step response of the closed-loop system using the ZN-PID controller with different time delay variations is shown in Fig. 11.

It can be seen clearly that, the system using proposed PID (Fig. 9) has better performance (smaller overshoot) and it is much more robust to the time delay variations than that using the ZN-PID controller.

Tuning rules of ISE-GPM-setpoint combines the advantages of designing both the gain and phase margin (GPM) and the time optimum design by minimizing the integral squared error (ISE) of the step setpoint response to give a set of optimal gain and phase margin tuning rules. The method performs four equations of gain margin, phase margin, unity magnitude at the gain cross-over frequency, and phase value of $-180^{\circ}$ at the phase crossover frequency. These four equations have five unknowns (PID gains and cross-over frequencies). The extra degree of freedom is used to achieve the minimum ISE. Curve fitting with the least-squares is used to estimate the tuning rules.

The PID controller gains for ISE-GPM-setpoint method are:

$$
\begin{aligned}
K_{p} & =\frac{1.8578}{K} A_{m}^{-0.9087} \theta_{m}^{0.0821}(L / T)^{-0.9471}=2.1397, \\
K_{i} & =K_{p} / T_{i}=3.1206, \text { where } \\
T_{i} & =\frac{0.0211 T\left(1+0.3289 A_{m}+6.4572 \theta_{m}+25.1914(L / T)\right.}{1+0.0625 A_{m}-0.8079 \theta_{m}+0.347(L / T)}, \\
K_{d} & =K_{p} T_{d}=0.2773, \text { where } T_{d}=0.4899 T A_{m}^{-0.0845} \theta_{m}^{0.1457}(L / T)^{1.0264}
\end{aligned}
$$

where $A_{m}=3$ and $\theta_{m}=56.8^{\circ} * \pi / 180$ are obtained from the proposed controller specification shown in Fig. 8.

The unit step response of the closed-loop system using the ISE-GPM-setpoint controller with different time delay variations is shown in Fig. 12.

It can be seen clearly that, the system using our proposed PID (Fig. 9) has better performance (smaller overshoot and settling time) and it is more robust to the time delay variations than that using the ISE-GPM-setpoint controller.

We measured the degree of robustness by computing the difference between the integral squared error (ISE) with 100\% time delay case and the ISE with the other two cases (80\% and $120 \%$ time delay). The parameters of PID controllers designed using the three tuning methods are presented in Table 1 . and the simulation results are presented in Table 2. 
Table 1.

Parameters of PID controllers designed using the three tuning methods.

\begin{tabular}{|c|c|c|c|}
\hline Method & $\boldsymbol{K}_{\boldsymbol{p}}$ & $\boldsymbol{K}_{\boldsymbol{i}}$ & $\boldsymbol{K}_{\boldsymbol{d}}$ \\
\hline Proposed method & 1.117 & 1.4238 & -0.11 \\
ZN & 3.5341 & 6.5299 & 0.4782 \\
ISE-GPM-setpoint & 2.1397 & 3.1206 & 0.2773 \\
\hline
\end{tabular}

Table 2.

Performance and robustness summary using the three tuning methods

\begin{tabular}{|c|c|c|c|c|}
\hline \multirow{2}{*}{ Method } & \multicolumn{4}{|c|}{ Simulation results } \\
\cline { 2 - 5 } & $\begin{array}{c}\text { Overshoot }(\%) \\
\text { with L = 0.3 sec }\end{array}$ & $\begin{array}{c}\text { Settling time } \\
\text { with L = } 0.3 \mathrm{sec}\end{array}$ & $\begin{array}{c}\text { (ISE with 100\% } \\
\text { L - ISE with } \\
80 \% \mathrm{~L})\end{array}$ & $\begin{array}{c}\text { (ISE with 120\% } \\
\text { L - ISE with } \\
100 \% \mathrm{~L})\end{array}$ \\
\hline Proposed & $5.18 \%$ & $2.7102 \mathrm{sec}$ & 0.5403 & 0.6151 \\
ZN & $52.0896 \%$ & $2.3200 \mathrm{sec}$ & 1.2024 & 2.0218 \\
ISE-GPM-SP & $18.3958 \%$ & $2.7503 \mathrm{sec}$ & 0.5932 & 0.8399 \\
\hline
\end{tabular}

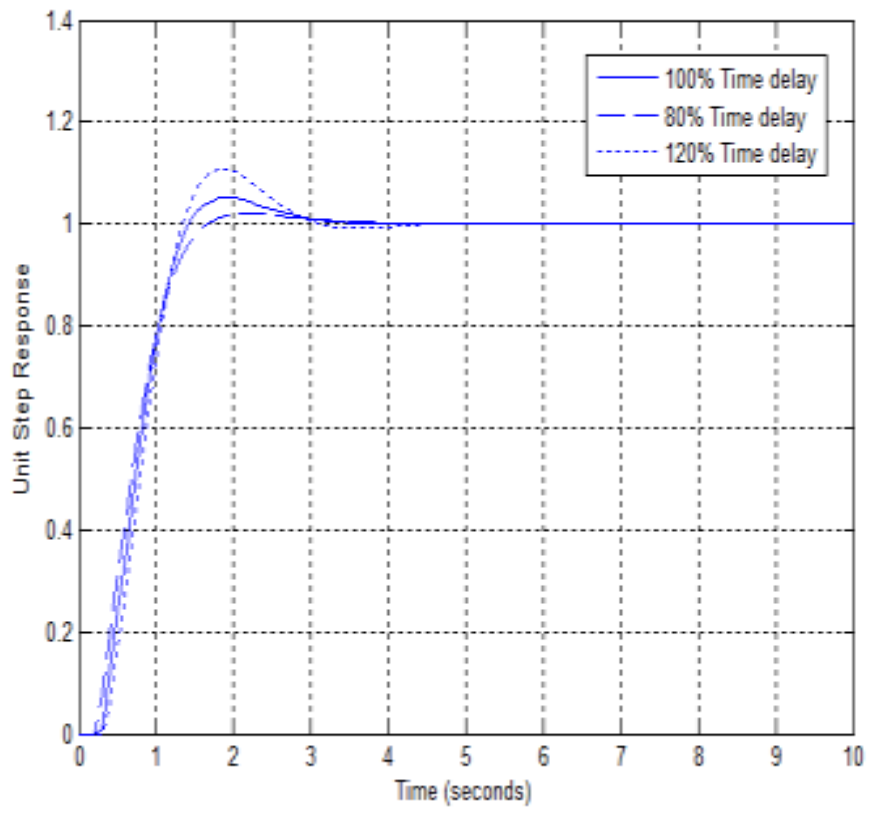

Fig. 9. Unit step responses with the proposed PID controller $G_{c-p r o p o s e d}(s)$. 


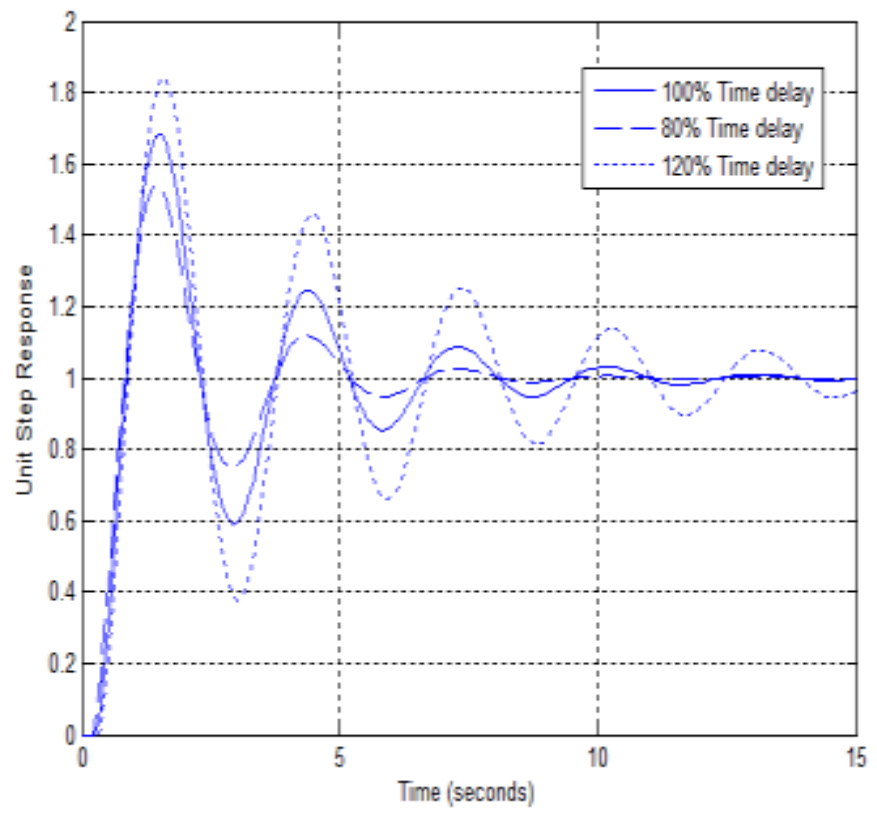

Fig. 10. Unit step responses with PID controller $G_{c 0}(s)$

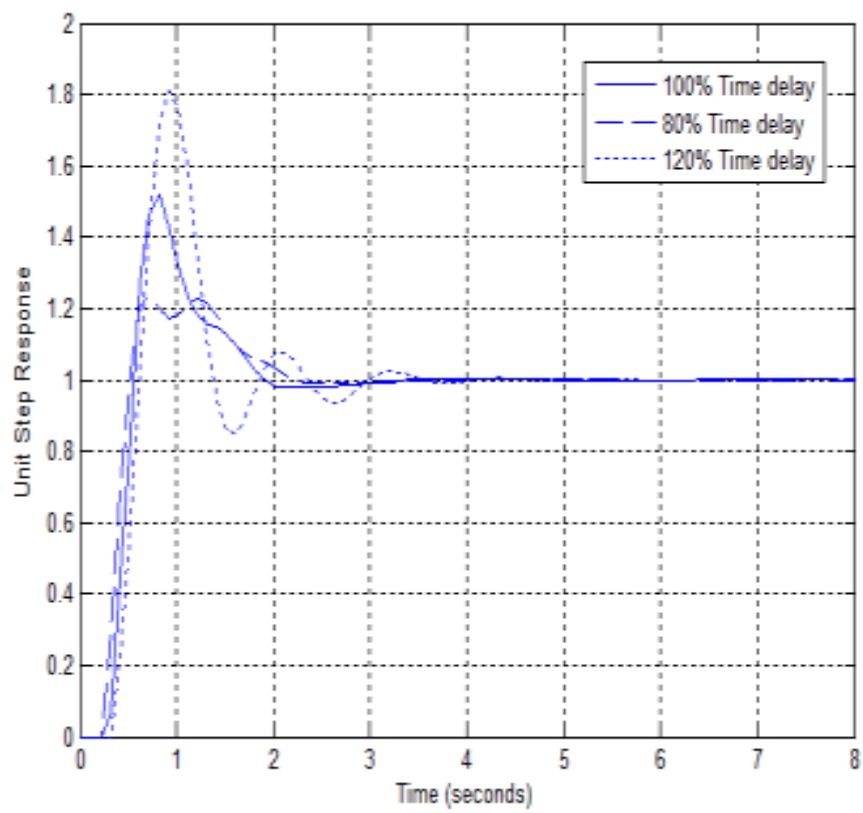

Fig. 11. Unit step responses with ZN-PID controller. 


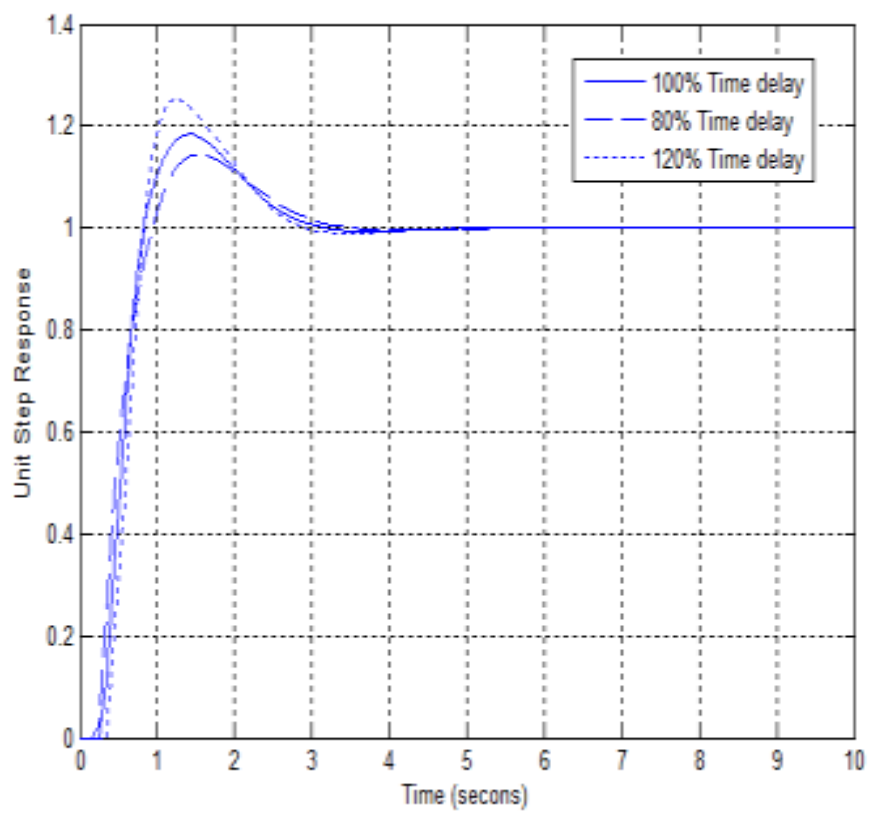

Fig. 12. Unit step responses with ISE-GPM-setpoint controller.

\section{Conclusion}

This paper considers the design method for PID controllers to achieve the robustness to the uncertainty of the time delay for the first-order plus time delay system (FOPTD). We first determine the stabilizing regions of the PID controller gains by a graphical stability method. Then, we specify two simultaneous design specifications: gain margin and phase crossover frequency. To get a unique PID controller, an additional constraint on the magnitude slope is introduced, by searching for the smallest absolute value of the derivative of the open-loop system magnitude with respect to the frequency at the specified phase crossover frequency. The obtained PID controller is located in the stability region, and also robust to system time delay variation due to that constraint.

The design steps of the proposed method are summarized in an example. It is shown in the illustrative example that the proposed method gives better performance (smaller overshoot) for the unit step response than that in the ZN method and GPM-ISE method. Also, the proposed PID controller is more robust to a bounded variation in the system time delay than that using ZN and GPM-ISE controllers.

\section{REFERENCES}

[1] K. Astrom and T. Hagglund, PID Controllers: Theory, Design, and Tuning, Research Triangle Park: Instrument Society of America, 1995.

[2] K. Astrom and T. Hagglund, Advanced PID Control, Research Triangle Park: Instrument Society of America, 2005.

[3] J. G. Ziegler and N. Nichols, "Optimum settings for automatic controllers", Transactions of the ASME, vol. 64, no. 8, pp. 759-768, 1942.

[4] S. P. Bhattacharyya, A. Datta, and L. H. Keel, Linear Control Theory: Structure, Robustness and Optimization. Taylor \& Francis Group, 2009. 
Noha Medhat Darwish, Design of robust PID controllers for first-order plus time delay ..........

[5] A. I. Saleh, M. M. Hasan, and N. M. Darwish, "Determination of Controllers Gains Limit Using The Mikhailov Stability Criterion", Journal of Engineering Sciences, Assiut University, Egypt, vol. 38, no. 1, pp. 209-219, 2010.

[6] G. J. Silva, A. Datta A, and S. P. Bhattacharyya, PID Controllers for Time-Delay Systems. Boston: Birkhäuser, 2005.

[7] N. Tan and I. Kaya, "Computation of stabilizing PI and PID controllers", In Proceedings of IEEE Conference on Control Applications, vol. 2, pp. 876-881, 2003.

[8] N. Tan, I. Kaya, C. Yeroglu, et al. "Computation of stabilizing PI and PID controllers using the stability boundary locus", Energy Conversion and Management, vol. 47, no. (18-19), pp. 3045-3058, 2006.

[9] Y. Luo and Y.Q. Chen, "Synthesis of robust PID controllers design with complete information on pre-specifications for the FOPTD systems", In Proceedings of American Control Conference, pp. 5013-5018, 2011.

[10] G. J. Silva, A. Datta A, and S. P. Bhattacharyya, "New Results on the Synthesis of PID Controller", IEEE Transaction on Automatic Control, vol. 47, no. 9, pp. 241-252, 2002.

[11] D. J. Wang, "Further results on the synthesis of PID controllers", IEEE Transaction on Automatic Control, vol. 52, no. 6, pp. 1127-1132, 2007.

[12] L. S. Pontryagin," On the zeros of some elementary transcendental function (in English)", American Mathematical Society Translation, vol. 2, pp. 95-110, 1955.

[13] W. K. HO, K. W. LIM, and W. XU, "Optimal Gain and Phase Margin Tuning for PID Controllers", Automatica, vol.34, no. 8, pp. 1009-1014, 1998.

[14] K. Li, "PID Tuning for Optimal Closed-Loop Performance With Specified Gain and Phase Margins", IEEE Transaction on Control Systems Technology, vol. 21, no. 3, pp. 10241030, 2013.

[15] Y. Luo and Y.Q. Chen, "Stabilizing and robust fractional order PI controller synthesis for first order plus time delay systems", Automatica, vol. 48, no. 9, pp. 2159-2167, 2012.

[16] J. Qibing, L. Qie, W. Qi, et al, "PID Controller Design Based on the Time Domain Information of Robust IMC Controller Using Maximum Sensitivity", Chinese Journal of Chemical Engineering, vol. 21, no. 5, pp. 529-536, 2013.

[17] C. H. Chang and K. W. Han, "Gain margins and phase margins for control systems with adjustable parameters", Journal of Guidance, Control, and Dynamics, vol. 13, no. 3, pp. 404-408, 1990.

[18] S. E. Hamamci, "An algorithm for stabilization of fractional-order time delay systems using fractional-order PID controllers", IEEE Transaction on Automatic Control, vol. 52, no. 10, pp. 1964-1969, 2007.

[19] J. Ackermann and D. Kaesbauer, "Stable polyhedral in parameter space", Automatica, vol. 39, no. 5, pp. 937- 943, 2003.

[20] S. E. Hamamci, and N. Tan, "Design of PI controllers for achieving time and frequency domain specifications simultaneously", ISA Transaction, vol. 45, no. 4, pp. 529-543, 2006. 


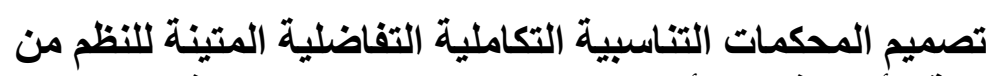
الدرجة الأولى ذات التأخير الزمنى بناء على خصائص فلى مجال التردد

الملخص العربى:

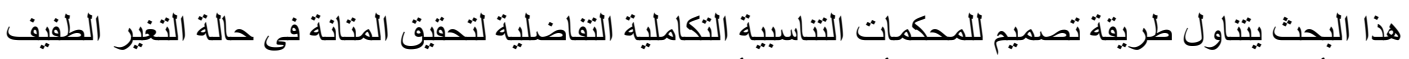
فى التأخير الزمنى للنظام من الدرجة الأولى ذو التأخير الزمنى. بداية تم ايجاد معاملات المحكمات التهات التناسبية

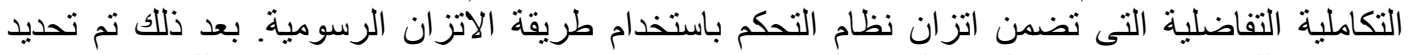

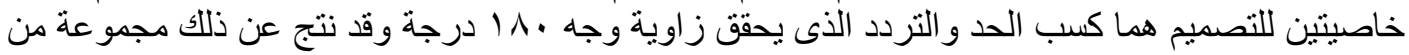
المحكمات التناسبية التكاملية التفاضلية التى تحقق قيم هاتين الخاصيتين. لايجاد محكم تناسبى تكاملى تفاضلى التى

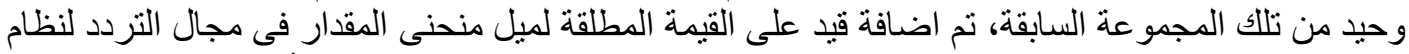

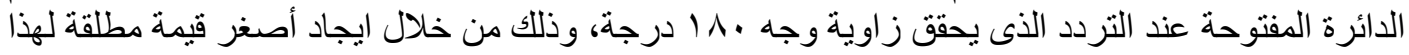

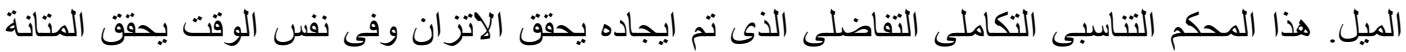
لنظام التحكم فى حالة التغير الطفيف فى التأخير الزمنى. النى التئ. 